\title{
Proceso Enfermero en aplicado a paciente con Depresión Mayor [F-32]: Intervenciones Secundarias
}

\section{Nursing Process applied to a patient with Major Depression [F-32]: Secondary Interventions.}

\author{
Ana P. Ortega-López ${ }^{a}$, José A. Ramírez-Ramírez ${ }^{b}$, Lizeth Rodríguez-Cruz $^{c}$, Roció E. Tello- \\ Sánchez ${ }^{d}$, Olga Rocio Flores Chávez ${ }^{e}$, Cristian A. Revoreda-Montes ${ }^{f .}$
}

\begin{abstract}
:
Depression is a health and disability problem in the population, an increase in the numbers of people who suffer from it has been observed in recent years, since it is a mental health pathology that has not been given the necessary relevance during its diagnosis, treatment and follow-up in the health, social and educational fields that is required. That is why the nursing process (NP) represents a fundamental methodological axis of nursing to provide care that will be necessary to implement in a specialized and individualized way in the patient with Major Depression Disorder (MDD).
\end{abstract}

Keywords:

Major depression, Mental health, Nursing, Nursing process

\section{Resumen:}

La depresión es un problema de salud y discapacidad en la población, se ha observado un incremento en las cifras de las personas que la padecen durante los últimos años, ya que es una patología en salud mental que no se le ha dado la relevancia necesaria durante su diagnóstico, tratamiento y seguimiento en los ámbitos sanitario, social y educativo que se requiere. Es por ello que el proceso enfermero (PE) representa un eje metodológico fundamental de enfermería para prestar cuidados que serán necesarios implementar de manera especializada e individualizada en el paciente con Trastorno de Depresión Mayor (TDM).

\section{Palabras Clave:}

Depresión mayor, Salud mental, Enfermería, Proceso enfermero

\section{Introducción}

La depresión se caracteriza por la presencia persistente de tristeza y una pérdida de interés en actividades que las personas normalmente disfrutan, acompañada de una incapacidad para llevar a cabo las actividades diarias, durante 14 días o más; dependiendo del número y gravedad de los síntomas, en función de la severidad se clasifica como leve, moderada o grave. En su forma más servera, la depresión puede conducir al suicidio (OMS, 2017).

\footnotetext{
a Universidad Autónoma del Estado de Hidalgo, https://orcid.org/https://orcid.org/ 0000-0002-0087-4598, Email: or391721@uaeh.edu.mx

${ }^{\mathrm{b}}$ Universidad Autónoma del Estado de Hidalgo, https://orcid.org/ https://orcid.org/ 0000-0002-9585-4660, Email: ra347057@uaeh.edu.mx c Universidad Autónoma del Estado de Hidalgo, https://orcid.org/https://orcid.org/0 0000-0001-7070-2321, Email: ro392618@uaeh.edu.mx

d Universidad Autónoma del Estado de Hidalgo, https://orcid.org/0000-0003-0576-9916, Email: rocio_tello9776@uaeh.edu.mx

e Universidad Autónoma del Estado de Hidalgo, https://orcid.org/ 0000-0001-9479-9834, Email: ofloresc@uaeh.edu.mx

Autor de Correspondencia, Universidad Autónoma del Estado de Hidalgo, https://orcid.org/0000-0001-5403-3527, Email: cristian_revoreda@uaeh.edu.mx
} 
La Organización Mundial de la Salud (OMS) estima que a nivel mundial hasta el año 2015 más de 300 millones de personas viven con depresión, es decir el $4,4 \%$ de la población mundial, observando un incremento de más del $18 \%$ entre 2005 y 2015 , por lo que es considerada la principal causa de problema de salud y discapacidad (OMS, 2017). El número de casos de trastorno depresivo (TD) en la región de las Américas es de 48.16 millones, lo que representa el $15 \%$ de la población total. Por otro lado, en nuestro país, se estimó un total de 4.936.614 de casos, lo que representa el $4,2 \%$ de la población total con este trastorno.

Las herramientas que integran los criterios de diagnóstico para la depresión mayor se encuentran determinados por El Manual Diagnóstico y Estadístico de los Trastornos Mentales (DSM-V) y la Clasificación Internacional de las Enfermedades (CIE-10), ambos basados en los síntomas que determinen la presencia o no del trastorno.

El Manual Diagnóstico y Estadístico de los Trastornos Mentales (DSM-V), menciona que el trastorno de depresión mayor (TDM) se caracteriza por episodios determinados de al menos dos semanas de duración que implican cambios claros en el afecto, la cognición y las funciones neurovegetativas, y remisiones interepisódicas., menciona los siguientes criterios diagnósticos:

A. Cinco (o más) de los síntomas siguientes han estado presentes durante el mismo periodo de dos semanas y representan un cambio del funcionamiento previo; al menos uno de los síntomas ya sea un estado de ánimo deprimido o perdida de interés o de placer.

1. Estado de ánimo deprimido la mayor parte del día, casi todos los días, según se desprende de la información subjetiva

2. Disminución importante del interés o el placer por todas o casi todas las actividades la mayor parte del día, casi todos los días (como se desprende de la información subjetiva o de la observación).

3. Pérdida importante de peso sin hacer dieta o aumento de peso, o disminución o aumento del apetito casi todos los días.

4. Insomnio o hipersomnia casi todos los días.

5. Agitación o retraso psicomotor casi todos los días

6. Fatiga o perdida de energía casi todos los días.

7. Sentimiento de inutilidad o culpabilidad excesiva 0 inapropiada (que puede ser delirante) casi todos los días
8. Disminución de la capacidad para pensar o concentrarse, o para tomar decisiones, casi todos los días.

9. Pensamientos de muerte recurrentes (no solo miedo a morir), ideas suicidas recurrentes sin un plan determinado, intento de suicidio o un plan específico para llevarlo a cabo.

B. Los síntomas causan malestar clínicamente significativo o deterioro en lo social, laboral u otras áreas importantes del funcionamiento.

C. El episodio no se puede atribuir a los efectos fisiológicos de una sustancia o de otra afección médica.

En la CIE10 el TDM se encuentra en el apartado de trastornos del estado de ánimo (afectivos) y se clasifica en: episodio depresivo leve, moderado, grave sin síntomas psicóticos, grave con síntomas psicóticos y otros tipos de episodios depresivos.

El trastorno de depresión mayor suele ser recurrente y, a menudo, el inicio de un episodio depresivo se relaciona con la presencia de acontecimientos o situaciones estresantes. 28

Dichas herramientas facilitan al equipo multidisciplinario realizar un diagnóstico oportuno y correcto del trastorno que se encuentra afectando a un individuo, por lo tanto, es de suma importancia que el personal de Enfermería en conjunto con la aplicación del Proceso Enfermero (PE), identifique los criterios diagnósticos que ayuden que el tratamiento y cuidados.

\section{Proceso Enfermero}

El proceso enfermero $(\mathrm{PE})$ representa el eje metodológico fundamental de enfermería para prestar cuidados. EI PE es un método sistemático y científico que permite realizar unos cuidados de enfermería efectivos e individualizados a las personas, familias y comunidades, con el fin de mantener recuperar un estado óptimo de salud. Se encuentra delimitado en cinco fases valoración, diagnóstico, planificación, ejecución y evaluación. 29

Dado que el cuidado es el objeto de estudio de enfermería, el PE ayuda a que este objeto de estudio se cumpla, ya que es un método sistemático a seguir por la enfermera (o), y brinda cuidados humanistas centrados en el logro de objetivos de manera eficaz, que permitirán organizar el trabajo y solucionar problemas relacionados con la salud de los individuos. EI PE está conformado de cinco etapas que obedecen a un orden lógico y conducen al logro de resultados, estas etapas son: valoración, diagnostico, planeación, ejecución y evaluación. Las cinco etapas se 
encuentran estrechamente relacionadas, de tal forma que el cumplimiento de una de ellas conduce a la siguiente. 30

1. La valoración nos permite reunir la información necesaria referente al usuario, familia y comunidad con el fin de identificar las respuestas humanas y fisiopatológicas, así como de recursos (capacidades) con los que se cuentan.

2. El diagnóstico consiste en el análisis de la información obtenida para emitir un juicio crítico sobre el estado de salud del usuario, familia y comunidad.

3. La planeación es el desarrollo de un proyecto donde se establecen objetivos y acciones encaminadas a predecir, prevenir y tratar problemas relacionados con la salud.

4. La ejecución consiste en llevar a la práctica el plan mediante acciones que conduzcan el logro de los objetivos establecidos.

5. La evaluación permite determinar el progreso del usuario, familia y comunidad hacia el logro de objetivos y valorar las competencias de la enfermera (o) en el desarrollo de las cuatro etapas anteriores, lo que posibilita hacer las modificaciones necesarias.

Se deberán implementar herramientas que ayuden a que el PE sea operativo en la práctica, así como agilizar los procesos y realizar un trabajo más autónomo y eficiente, dando respuestas al individuo y al grupo desde la evidencia científica y teniendo en cuenta la sostenibilidad del sistema, en la enfermería de práctica avanzada, en la gestión de casos en la continuidad de cuidados o como gestores de unidades de gestión clínica. En este sentido, los patrones funcionales de Mary Gordon es un método que permite a la enfermera (o), la identificación de patrones (secuencia de comportamientos) mediante la recolección de datos en forma sistemática y continua; está compuesto por 11 patrones funcionales de salud que a su vez se encuentran divididos en físicos y emocionales, además de implementar también el modelo de Análisis de Resultado del Estado Actual (modelo AREA) de Pesut; que establece redes de razonamiento clínico, basado en las relaciones que se establecen entre un número elevado de diagnósticos, ya que plantea una alternativa al razonamiento clínico tradicional. Muchos diagnósticos NANDA se relacionan entre sí, y lo podemos representar de forma gráfica a través de lo que se denomina red lógica. 29, 30

\section{Presentación del caso}

Paciente de 15 años, de Pachuca, Hidalgo, actualmente vive con sus padres, abuela paterna y su hermana menor de 12 años. Se encuentra cursando 30 semestre de bachillerato con disminución del rendimiento escolar en los últimos 6 meses y con una dificultad notable de socialización. Es referida al Servicio de Urgencias del Hospital General de Pachuca para valoración psiquiátrica por intento autolítico al precipitarse de un muro de 2 metros de altura mientras se encontraba con su hermana en el parque cercano a casa. No hay antecedentes somáticos de interés. Antecedentes psiquiátricos: Estudios previos por Neuropsiquiatría por presencia de rasgos de Trastorno del Espectro Autista (TEA), sin llegar a diagnóstico clínico. Actualmente a seguimiento por Salud Mental desde el año 2018 por estado de ánimo bajo recurrente, episodios de autolesión y alteración de la conducta en contexto de ansiedad. Tratamiento con Sertralina $50 \mathrm{mg}$.

\section{Etapa I: Valoración por patrones funcionales}

\section{Patrón I: Percepción y Control de la salud}

Sin antecedentes somáticos de interés, no refiere alergias conocidas. Completamente autónoma e independiente en las Actividades Básicas de la Vida Diaria (ABVD). Sin datos de toxicomanías.

Episodio depresivo actual a seguimiento por Salud Mental desde junio de 2018, actitud apática en relación con su patología, y desinterés acerca de la misma. No realiza ninguna crítica de lo sucedido. Tratamiento con Sertralina $50 \mathrm{mg} 1$ comprimido a la cena.

\section{Patrón II: Nutricional / metabólico}

No hay dificultad para la masticación ni para la deglución. Independiente para las ingestas. No presencia de náuseas, ni vómitos. Piel y mucosas hidratadas.

Actualmente refiere alteración del apetito por defecto, con ingestas escasas y apenas realizando 2 comidas principales al día (comida y cena), comenta que, por desgana, aunque en condiciones normales refiere comer de todo y variado.

IMC: $18,5 \mathrm{~kg} / \mathrm{m} 2$, al límite entre bajo y peso ideal.

\section{Patrón III: Eliminación}

Continencia urinaria y fecal.

Presencia de estreñimiento ocasional desde que comenzó con pérdida del apetito, aspecto normal.

\section{Patrón IV: Actividad/ejercicio}

Actualmente sólo realiza actividad física en el ámbito escolar. Ha abandonado recientemente el equipo al que pertenecía de fútbol por falta de energía y apatía. Refiere cansancio, aunque no realice ninguna actividad característica más que ir al instituto.

Tensión arterial (TA): 112/69 mm/Hg.

Frecuencia cardiaca (FC): 81 lpm. 


\section{Patrón V: Sueño/descanso}

Buena higiene del sueño, comenta dormir 9 horas nocturnas y 1 hora y media como mínimo diurna.

Con tendencia a clinofilia.

\section{Patrón VI: Cognitivo / perceptivo}

Consciente, orientada en las tres esferas y colaboradora. Abordable y tranquila durante la entrevista. Discurso coherente y fluido, así como bien dirigido.

\section{Patrón VII: Autopercepción / autoconcepto}

Refiere autoestima baja desde hace años, coincidiendo con el inicio de la adolescencia, así como sentimientos de inferioridad en comparación a sus compañeros de instituto e incluso con su hermana menor. Presencia de ideas de muerte, aunque sin estructuración.

\section{Patrón VIII: Función y relación}

Refiere buena relación con sus padres y su abuela, de la que se siente en muchas ocasiones responsable de su cuidado. Presencia de una relación más distante con su hermana, aunque con una buena dinámica familiar. Gran dificultad de socialización, refiere ser incapaz de realizar amistades. Escasas relaciones sociales, limitadas al transcurso escolar. Reconoce ser bastante retraída.

\section{Patrón IX: Sexualidad / reproducción}

No tiene pareja actualmente. Ciclos menstruales regulares.

\section{Patrón X: Afrontamiento / tolerancia al estrés}

Comenta grandes niveles de frustración a consecuencia de cómo es su día a día y la falta de mejoría, con consiguientes momentos de estrés y ansiedad que canaliza en forma de autolesiones en MMSS y MMII de poca gravedad. Dificultad en dichos momentos para el control de impulsos.

\section{Patrón XI: Valores y creencias}

A pesar del suceso ocurrido mantiene planes de futuro activo (quiere ser transportista). No comenta ninguna creencia religiosa o cultural que le gustaría que tuviésemos en cuenta.

\section{Etapa II: Diagnostico}

Dentro del Proceso Enfermero es primordial agrupar los datos significativos (evidencia) y posteriormente realizar una clasificación en datos objetivos y datos subjetivos, es importante el poder identificarlos adecuadamente debido a que serán una parte esencial y facilitarán la identificación de los diagnósticos de enfermería, para ello se utilizó la tabla de razonamiento diagnóstico y la NANDA 2018-2020 (11 ${ }^{\circ}$ edición). Encontrando lo siguientes diagnósticos:

1. Deterioro de la regulación del estado de ánimo relacionado con ansiedad, cambios en el apetito, deterioro del funcionamiento social, pensamientos recurrentes de muerte como lo demuestra actitud apática, desinterés, alteración del apetito por defecto, ingestas escasas, astenia. con la condición asociada propia de la enfermedad crónica, lo que la coloca en esta población de riesgo. Lo que implicará que para abordar este diagnóstico se requerirá de intervenciones colaborativas con otras disciplinas.

2. Afrontamiento ineficaz relacionado con estrategias de liberación de la tensión ineficaces, momento inadecuado para prepararse para el agente estresante, insuficiente sentido de control como lo demuestra autolesiones en MMSS y MMII de poca gravedad, fatiga, dificultad del control de impulsos, incapacidad para satisfacer las expectativas del rol, incapacidad para afrontar una situación, insuficiente resolución de los problemas, encontrándose en una crisis situacional lo que la coloca en una población de riesgo. Lo que implicará que para abordar este diagnóstico se requerirá de intervenciones colaborativas con otras disciplinas.

3. Ansiedad relacionada con amenaza de muerte, amenaza para el estado actual, necesidades no satisfechas como lo demuestra canaliza en forma de autolesiones en MMSS y MMII de poca gravedad, clinofilia, sentimiento de insuficiencia, alteración en la atención, ya que se encuentra en una crisis situacional, lo que la coloca en esta población de riesgo. Lo que implicará que para abordar este diagnóstico se requerirá de intervenciones colaborativas con otras disciplinas.

4. Fatiga relacionada con ansiedad, depresión, malnutrición como lo demuestra apatía, cansancio, deterioro de la habilidad para mantener las actividades habituales, desinterés por el entorno, energía insuficiente, patrón de sueño no reparador, con la condición asociada de fatiga pues se ha expuesto a acontecimientos vitales negativos, lo que la cataloga como población de riesgo. Lo que implica que para abordar este diagnóstico se requerirá de intervenciones colaborativas con otras disciplinas.

5. Desequilibrio nutricional: ingesta inferior a las necesidades relacionado con ingesta insuficiente de alimentos como lo demuestra falta de interés en la comida, ingesta de alimentos inferior a las cantidades diarias recomendadas, con la condición asociada propia del trastorno psicológico y los factores biológicos, lo que la coloca en esta población de riesgo. Lo que implicará que para abordar este diagnóstico se requerirá de intervenciones colaborativas con otras disciplinas. 
6. Automutilación relacionada con baja autoestima, estrategias de afrontamiento ineficaz, impulsividad, impulso irresistible de autolesionarse, sentimientos negativos como lo demuestra autolesiones en MMSS y MMII de poca gravedad, intento autolítico, episodios anteriores de autolesión, con la condición asociada propia del trastorno psicótico ya que se encuentra en la adolescencia y tiene historia previa de violencia autodirigida, lo que la coloca en esta población de riesgo. Lo que implicará que para abordar este diagnóstico se requerirá de intervenciones colaborativas con otras disciplinas, con la condición asociada propia del trastorno psiquiátrico, y presenta antecedentes de riesgo suicida, lo que la coloca en población de riesgo. Lo que implicará que para abordar este diagnóstico se requerirá de intervenciones colaborativas con otras disciplinas.

7. Riesgo de suicidio evidenciado por cambios notables en el desempeño escolar, cambios notables en la actitud, cambios notables en la conducta. Lo que implicará que para abordar este diagnóstico se requerirá de intervenciones colaborativas con otras disciplinas.

A partir del diagnóstico enfermero se jerarquizaron los diagnósticos con el uso del modelo AREA mediante el uso de la Red Lógica del Modelo de Análisis del Resultado del Estado Actual (Figura No.1).

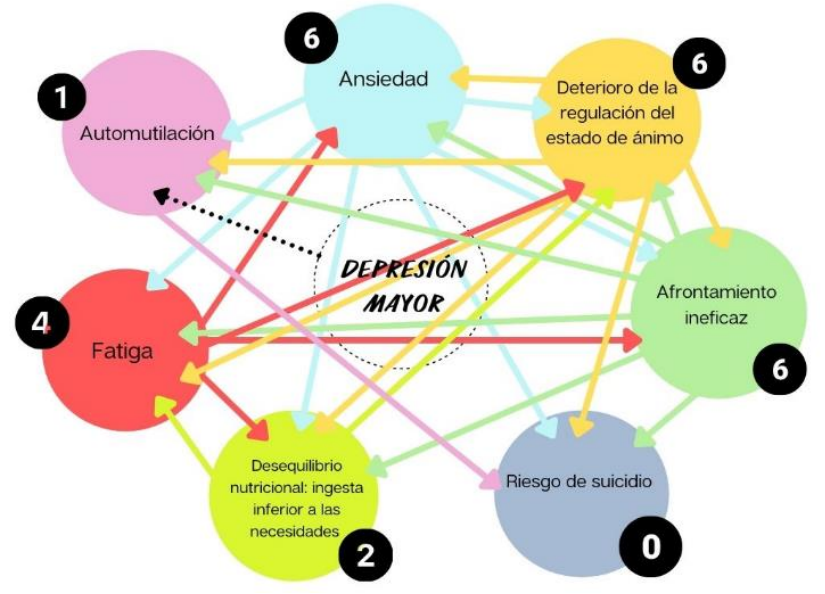

Figura No. 1 Jerarquización de los diagnósticos NANDA

Con base a la utilización del modelo AREA, además de aplicar la premisa vida-función-estética dentro del proceso enfermero, los diagnósticos principales quedan de la siguiente manera:

1. Deterioro de la regulación del estado de ánimo relacionado con ansiedad, cambios en el apetito, deterioro del funcionamiento social, pensamientos recurrentes de muerte como lo demuestra actitud apática, desinterés, alteración del apetito por defecto, ingestas escasas, astenia.

2. Afrontamiento ineficaz relacionado con estrategias de liberación de la tensión ineficaces, momento inadecuado para prepararse para el agente estresante, insuficiente sentido de control como lo demuestra autolesiones en MMSS y MMII de poca gravedad, fatiga, dificultad del control de impulsos, incapacidad para satisfacer las expectativas del rol, incapacidad para afrontar una situación, insuficiente resolución de los problemas.

3. Ansiedad relacionada con amenaza de muerte, amenaza para el estado actual, necesidades no satisfechas como lo demuestra canaliza en forma de autolesiones en MMSS y MMII de poca gravedad, clinofilia, sentimiento de insuficiencia, alteración en la atención.

\section{Etapa III y IV: Planeación y Ejecución}

En esta etapa se establecieron las estrategias necesarias para reforzar las respuestas humanas encontradas en la etapa de valoración, y estas determinan cómo se brindarán los cuidados de enfermería a través del Proceso Enfermero. Para la implementación de estas intervenciones se requiere la colaboración de distintos enfoques multidisciplinarios. A partir de la evaluación de las principales respuestas humanas, así como el establecimiento de las prioridades secundarias (diagnostico secundario), basándose en la evidencia, los estándares relativos a la salud mental y la psiquiatría; se determinaron los problemas urgentes, y después los problemas generales. Para con base en estos datos, se estableciera el objetivo (NOC) a lograr en la paciente; mismo que dirigirá las intervenciones, así como los que son factores de protección para de la paciente.

Para el diagnostico de Afrontamiento ineficaz relacionado con estrategias de liberación de la tensión ineficaces, momento inadecuado para prepararse para el agente estresante, insuficiente sentido de control de impulsos como lo demuestran las autolesiones en MMSS y MMII, fatiga, dificultad del control de impulsos, incapacidad para satisfacer las expectativas del rol, incapacidad para afrontar una situación e insuficiente resolución de los problemas.

Se estableció el resultado NOC, para medir la resolución del diagnóstico enfermero: Equilibrio emocional, perteneciente a al Domino III: Salud Psicosocial, y a la Clase M: Bienestar psicológico. Para ello se establecieron los siguientes indicadores:

1. Muestra un afecto adecuado a la situación

2. Muestra control de sus impulsos

3. Refiere apetito normal

4. Muestra interés por lo que le rodea 


\section{Ideas suicidas}

6. Depresión

Con la escala de medición: Nunca demostrado 1, Raramente demostrado 2, A veces demostrado 3, Frecuentemente demostrado 4, siempre demostrado 5 y Siempre demostrado 1, frecuentemente demostrado 2, A veces demostrado 3, Raramente demostrado 4, Nunca demostrado, respectivamente.

A partir del establecimiento del objetivo se plantearon las siguientes intervenciones

Entrenamiento para controlar los impulsos

CAMPO: 3 . Conductual

CLASE: O. Terapia conductual

Nota: para esta intervención se requiere de la colaboración de un especialista en salud mental o psiquiatría.

-Ayudar al paciente a identificar el problema 0 situación que requiera una acción inmediata. Revisión sistemática, GPC, Depresión Mayor en la Infancia y Adolescencia, 2018. Factores de riesgo y protectores, GPC, Depresión Mayor en la Infancia y Adolescencia, 2018.

-Utilizar un plan de modificación de la conducta que sea apropiado para reforzar la estrategia de solución de problemas que se hayan enseñado. El abordaje terapéutico, GPC, Depresión Mayor en la Infancia y Adolescencia, 2018.

- Seleccionar la estrategia de solución de problemas adecuada al nivel de desarrollo y la función cognoscitiva del paciente. El abordaje terapéutico, GPC, Depresión Mayor en la Infancia y Adolescencia, 2018.

- Enseñar al paciente a "detenerse y pensar" antes de comportarse impulsivamente. Intervenciones psicológicas tempranas basadas en el manejo de las emociones, Mamani-Benito, Brousett-Minaya, CcoriZúñiga, \& Villasante-ldme, 2018. El abordaje terapéutico, GPC, Depresión Mayor en la Infancia y Adolescencia, 2018.

- Ayudar al paciente a evaluar el resultado del curso de acción elegido. Estrategias diagnósticas y terapéuticas, GPC, Depresión Mayor en la Infancia y Adolescencia, 2018.

- Animar al paciente a recompensarse a sí mismo por los resultados exitosos, Terapia Cognitivo Conductual, Terapia Dialecto Conductual, GPC de Prevención y Tratamiento de la Conducta Suicida, 2020.

- Animar al paciente a practicar la solución de problemas en situaciones sociales e interpersonales, fuera del ambiente terapéutico seguido por la evaluación de resultados. Terapia de resolución de problemas (TRP), Terapia Cognitivo Conductual, GPC de Prevención y Tratamiento de la Conducta Suicida, 2020.

Escucha Activa

Campo: 3. Conductual
Clase: Q. Potenciación de la comunicación

-Mostrar interés por el paciente. Tratamiento psicológico, GPC Manejo de Pacientes con Trastornos de Ansiedad en Atención Primaria.

-Centrarse completamente en la interacción eliminando prejuicios, sesgos, presunciones, preocupaciones personales y otras distracciones. Tratamiento no farmacológico, GPC Diagnóstico y tratamiento de los trastornos de ansiedad del adulto.

-Mostrar conciencia y sensibilidad hacia las emociones. Tratamiento no farmacológico, GPC Diagnóstico y tratamiento de los trastornos de ansiedad del adulto. El abordaje terapéutico, GPC, Depresión Mayor en la Infancia y Adolescencia, 2018.

-Estar atento al tono, ritmo, volumen, entonación e inflexión de la voz. Tratamiento no farmacológico, GPC Diagnóstico y tratamiento de los trastornos de ansiedad del adulto.

-Identificar los temas predominantes. (Información/comunicación al paciente Guía de Práctica Clínica para el Manejo de Pacientes con Trastornos de Ansiedad en Atención Primaria ,2008).

-Verificar la comprensión del mensaje mediante el uso de preguntas y retroalimentación. (Tratamiento psicológico, Guía de Práctica Clínica para el Manejo de Pacientes con Trastornos de Ansiedad en Atención Primaria ,2008).

-Evitar barreras a la escucha activa (minimizar sentimientos, ofrecer soluciones sencillas, interrumpir, hablar de uno mismo y terminar de manera prematura). (El abordaje terapéutico, GPC, Depresión Mayor en la Infancia y Adolescencia, 2018.)

-Utilizar el silencio/escucha para animar a expresar sentimientos, pensamientos y preocupaciones. Terapia de solución de problemas, (Manejo de la Depresión en el Adulto, GPC, 2010).

Mejorar el afrontamiento

Campo: 3. Conductual

Clase: R. Ayuda para el afrontamiento

-Utilizar un enfoque sereno, de reafirmación. Terapia de solución de problemas GPC, Manejo de la Depresión en el Adulto

-Disponer un ambiente de aceptación. Terapia cognitivo-conductual GPC, Manejo de la Depresión en el Adulto

-Evaluar la capacidad del paciente para tomar decisiones. Algoritmo 2.GPC Diagnóstico y Tratamiento del Trastorno depresivo en el adulto. Inventario de estrategias de afrontamiento, Jauregui, HerreroFernández \& Estévez, 2016.

-Tratar de comprender la perspectiva del paciente sobre una situación estresante. Terapia cognitivoconductual GPC, Manejo de la Depresión en el Adulto. Algoritmo terapéutico para la depresión mayor leve, 
moderada y grave, GPC, Depresión Mayor en la Infancia y Adolescencia, 2018

-Animar al paciente a desarrollar relaciones. El abordaje terapéutico, GPC, Depresión Mayor en la Infancia y Adolescencia, 2018.

-Favorecer las relaciones con personas que tengan intereses y objetivos comunes. El abordaje terapéutico, GPC, Depresión Mayor en la Infancia y Adolescencia, 2018.

-Alentar la manifestación de sentimientos, percepciones y miedos. Algoritmo terapéutico para la depresión mayor leve, moderada y grave, GPC, Depresión Mayor en la Infancia y Adolescencia, 2018.

-Animar al paciente a identificar sus puntos fuertes y sus capacidades. El abordaje terapéutico, GPC, Depresión Mayor en la Infancia y Adolescencia, 2018.

\section{Etapa V: Evaluación}

En esta etapa se valoró cuidadosa y detalladamente los aspectos que ocurrieron durante todo el proceso enfermero, desde la valoración hasta la implementación propia de las intervenciones y la delegación de las acciones, lo que ha dictado continuar con el proceso; para esto se realizó una evaluación preintervención mediante los indicadores NOC, encontrando una puntuación Diana de 2, y se estableció la posibilidad de aumentar a 3, manteniendo en todo momento la puntación en un mínimo de 2 puntos.

\begin{tabular}{|l|l|l|l|l|l|}
\hline Indicadores & $\mathbf{1}$ & $\mathbf{2}$ & $\mathbf{3}$ & $\mathbf{4}$ & $\mathbf{5}$ \\
\hline Muestra un afecto adecuado a la situación & & $\mathbf{X}$ & & & \\
\hline Muestra control de sus impulsos & & $\mathbf{X}$ & & & \\
\hline Refiere apetito normal & & $\mathbf{X}$ & & & \\
\hline Muestra interés por lo que le rodea & & $\mathbf{X}$ & & & \\
\hline Ideas suicidas & & $\mathbf{X}$ & & \\
\hline Depresión & $\mathbf{x}$ & & & \\
\hline
\end{tabular}

Una vez ejecutadas las intervenciones se realizó una evaluación pos-intervención encontrando una puntuación Diana de 3, lo que sugiere continuar con el Proceso Enfermero, colocando como meta aumentar una puntuación diana de al menos 4 , siempre manteándola a 3 puntos al menos.

\begin{tabular}{|c|c|c|c|c|c|}
\hline \multicolumn{6}{|l|}{ Indicadores } \\
\hline & 1 & 2 & 3 & 4 & 5 \\
\hline Muestra un afecto adecuado a la situación & & & $\mathbf{x}$ & & \\
\hline Muestra control de sus impulsos & & & $\mathbf{x}$ & & \\
\hline Refiere apetito normal & & & $\mathbf{x}$ & & \\
\hline Muestra interés por lo que le rodea & & & $\mathbf{x}$ & & \\
\hline Ideas suicidas & & & $\mathbf{x}$ & & \\
\hline Depresión & & $\mathbf{x}$ & & & \\
\hline
\end{tabular}

\section{Conclusión}

Sin lugar a dudas el PE, mejora de manera sustancial la calidad de los cuidados de Enfermería en cualquier área asistencial, para este caso la Intensidad de Enfermería fue 145 minutos lo que contribuyó a satisfacer las necesidades y mejorar la funcionalidad de cada patrón, y permitió garantizar el bienestar, la satisfacción usuaria y la confianza en el actuar específico del personal de enfermería. EI PE pone de manifiesto que, su uso no discrimina el uso y la vinculación de las GPC, los AAC, las Acciones Esenciales para la Seguridad del Paciente y las Metas Internacionales, por el contrario, se cimenta la Práctica Basada en Evidencias además que permite implementar de forma correcta los cuidados, optimizar los recursos de diagnóstico y tratamiento, así como la referencia y contra referencia a los niveles de atención correspondiente. Además de que se debe fomentar el empoderamiento de enfermería, el PE nos brinda la oportunidad de unir los conocimientos, habilidades y destrezas que se adquieren a lo largo de la formación académica, recordar que cada una de las etapas es importante, ya que al realizar una de ellas está nos conducirá a la siguiente, es importante manejar y elaborar cuidadosamente cada una de ellas para obtener un proceso organizado y especializado que busca el beneficio máximo del paciente de manera individualizada.

\section{Recomendaciones}

Para el manejo de la depresión se recomienda promover mediante la escucha activa la comunicación entre enfermero-paciente acerca de las emociones y sentimientos. Así como recordar que una valoración deberá ser sistemática y focalizada de manera adecuada. Se deben de incluir intervenciones psicológicas, farmacológicas y psicosociales durante el tratamiento de la depresión en la infancia y en la adolescencia, con un enfoque integral que permita mejorar el bienestar y la capacidad funcional del paciente. Además, es de suma importancia que en el manejo de la depresión se incluyan siempre los cuidados de buena práctica clínica, que con base a la GPC sobre la Depresión Mayor en la Infancia y Adolescencia, menciona tales como: el seguimiento regular y frecuente del paciente, la psicoeducación enfocada en la familia y comunidad, así como el apoyo y cuidado individual y familiar, técnicas psicoterapéuticas básicas, la atención a comorbilidades y la coordinación con otros profesionales (ámbito sanitario, social y educativo). Deberá prestarse atención ante la presencia de factores familiares y sociales que puedan interferir en la evolución favorable del TDM, y en caso indicar su tratamiento $y / 0$ establecer las medidas pertinentes para la disminución de su daño o resolución.

\section{Referencias}


Guiasalud.es.

Available

from: https://portal.guiasalud.es/wpcontent/uploads/2018/12/GPC_575_Dep resion_infancia_Avaliat_compl.pdf

[2] Grupo de trabajo de la Guía de Práctica Clínica de Prevención y Tratamiento de la Conducta Suicida. Plan de Calidad para el Sistema Nacional de Salud del Ministerio de Sanidad, Política Social e Igualdad. Axencia de Avaliación de Tecnoloxías Sanitarias de Galicia (avalia-t); 2010.Guías de Práctica Clínica en el SNS: Avalia-t 2010/02.

[3] Yanchaguano Zamora CE. Adicción al internet y autoestima en adolescentes: Unidad Educativa Fiscomisional La Inmaculada. Quito: UCE; 2019.

[4] Figueroa-Contreras, J. La Autoestima en el Rendimiento Escolar de los estudiantes del tercer año de educación básica de la escuela "Enrique gil Gilbert" de Guayaquil. Diss. 2012.

[5] Ceballos-Ospino GA, Suarez-Colorado Y, Suescún-Arregocés J, Gamarra-Vega LM, González KE, Sotelo-Manjarres AP. Ideación suicida, depresión y autoestima en adolescentes escolares de Santa Marta. Duazary. 2015;12(1):15.

[6] Mamani-Benito OJ, Brousett-Minaya MA, Ccori-Zúñiga DN Villasante-Idme KS. La inteligencia emocional como factor protector en adolescentes con ideación suicida. Duazary. 2018.

[7] Aviles Saez Z, Cara Rodríguez R, López Trinidad LM. Comunicación del personal de Enfermería con el paciente con patología psiquiátrica = Nursing staff communication with psychiatric pathology patient. Rev esp comun salud. 2018.

[8] Márquez MO. Depresión y Calidad de la Dieta. iMedPub Journals [Internet]. 10032016; Disponible en https://www.archivosdemedicina.com/medicina-de-familia/depresiny-calidad-de-la-dieta-revisinbibliogrfica.pdf

[9] Cara Rodríguez R, Avilés Sáez Z, López Trinidad LM. Comunicación y escucha activa por parte del profesional de Enfermería a pacientes con cáncer ginecológico: una revisión bibliográfica $=$ Nursing staff communication and active listening to patients with gynecological cancer: a bibliographic review. Rev esp comun salud. 2018.

[10] Lillo y Julio Antonio Guija AMMJMR. LOS TRASTORNOS DEL CONTROL DE LOS IMPULSOS Y LAS PSICOPATÍAS: Psiquiatría y Ley. C/ Arturo Soria, no 311, 1o B 28033 Madrid (España: Fundación Española de Psiquiatría y Salud Mental; 2015

[11] Macías MA, Madariaga Orozco C, Valle Amarís M, Zambrano J. Estrategias de afrontamiento individual y familiar frente a situaciones de estrés psicológico. psicol desde caribe. 2016;30(1):123-45.

[12] Aitziber Pascual Jimeno SCL. Regulación emocional y afrontamiento: Aproximación conceptual y estrategias. Revista Mexicana de Psicología 2019;36(1):74-83,. Disponible en https://www.redalyc.org/jatsRepo/2430/243058940007/html/index.ht $\mathrm{ml}$

[13] Martínez Garduño MD, Gómez Martínez V, Siles Juárez P. Apoyo emocional: conceptualización y actuación de enfermería; una mirada desde la perspectiva del alumno. Enferm univ [Internet]. 2018;6(3) Disponible en: http://dx.doi.org/10.22201/eneo.23958421e.2009.3.329

[14] Ibáñez-Aguirre C. Claves psicopatológicas de las conductas autoagresivas en la adolescencia. Revista de Psicología Clínica con Niños y Adolescentes. 2017;65-70.

[15] Tratamiento.Guía de Práctica Clínica de Prevención y Tratamiento de la Conducta Suicida. Plan de Calidad para el Sistema Nacional de Salud del Ministerio de Sanidad, Política Social e Igualdad. Axencia de Avaliación de Tecnoloxías Sanitarias de Galicia (avalia-t); 2020.Guías de Práctica Clínica en el SNS: Avalia-t 2020/09.

[16] Grupo de Trabajo de la Guía de Práctica Clínica para el Manejo de Pacientes con Trastornos de Ansiedad en Atención Primaria. Madrid: Plan Nacional para el SNS del MSC. Unidad de Evaluación de Tecnologías Sanitarias. Agencia Laín Entralgo. Comunidad de Madrid; 2008. Guías de Práctica Clínica en el SNS: UETS Nº 2006/10

[17] Lucía Ferrer, María Martín, David Pineda, Bonifacio Sandín, José A Piqueras. Relación de la ansiedad y la depresión en adolescentes con dos mecanismos transdiagnósticos: el perfeccionismo y la rumiación. REVISTA INTERNACIONAL DE PSICOLOGIA CLINICA Y SALUD. 2018;26(1):55-74.

[18] Carlos Díaz Santos LSV. La ansiedad en la adolescencia. RqR Enfermería Comunitaria, 2018;6(1):21-31.

[19] Diagnóstico y Tratamiento del Trastorno depresivo en el adulto. México: Secretaría de Salud; 1 de diciembre de 2015

[20] Diagnóstico y Tratamiento del Trastorno depresivo en el adulto en el adulto mayor en primer nivel de atención. Mexico: Secretaria de salud,2011.

[21] Herdman TH, Kamitsuru S. NANDA International Nursing Diagnoses: Definitions \& Classification, 2018-2020. 11a ed. Herdman TH, editor. New York, NY: Thieme Medical; 2017.

[22] Butcher HK, Bulechek GM, Dochterman JM, Wagner C, editores. Clasificación de Intervenciones de Enfermería (NIC). 7a ed. Elsevier; 2018.

[23] Moorhead S, Johnson M, Maas ML, editores. Clasificación de Resultados de Enfermería (NOC). 6a ed. Elsevier Health Sciences; 2019.

[24] Quintana E. El papel de enfermería en la depresión mayor. Escuela de Enfermería: “"Casa Salud Valdecilla"'. 2017

[25] Garza-Laguera, A. y Castro, C. Terapia Breve en un caso de Autolesión no suicida. Psicología Científica [Internet]. 2016;9(11). Disponible en: http://www.psicologiacientifica.com/terapia-breve-caso-autolesionno-suicida

[26] Bilbao PJ, Fernández DH, Gutiérrez AE. Estructura factorial del "Inventario de estrategias de afrontamiento" y su relación con la regulación emocional, ansiedad y depresión. Psicol Conductual. 2016;24(2):319-40.

[27] Alzuri Falcato M, Hernández Muñoz N, Calzada Urquiola Y. Depresión en la adolescencia: consideraciones necesarias para su diagnóstico y tratamiento. Finlay. 2017;7(3):152-4.

[28] Pérez-Padilla Elsy Arlene, Cervantes-Ramírez Víctor Manuel, Hijuelos-García Nayeli Alejandra, Pineda-Cortés Juan Carlos, Salgado-Burgos Humberto. Prevalencia, causas y tratamiento de la depresión Mayor. Rev. biomédica [revista en la Internet]. 2017 Ago [citado 2021 Jun 29]; 28(2): 73-98. Disponible en: http://www.scielo.org.mx/scielo.php?script=sci_arttext\&pid=S2007$84472017000200073 \& \operatorname{lng}=$ es. https://doi.org/10.32776/revbiomed.v2 $8 \mathrm{i} 2.557$.

[29] Roch JLG. Enfermería psiquiátrica. Elsevier; 2016.

[30] Rodríguez S. BA. PROCESO DE ENFERMERÍA APLICACIÓN ACTUAL ED.3. CUELLAR AYALA; 2015 
Publicación semestral, Educación y Salud Boletín Científico Instituto de Ciencias de la Salud Universidad Autónoma del Estado de Hidalgo, Vol. 10, No. 19 (2021) 151-159

[31] Iglesias, JA. “¿Hacia dónde va el proceso enfermero de la tercera generación?" RevistaEnfermeríaCyL 9.2 (2017): 1-2.

[32] Sevilla, Juan Carlos R. "Papel de enfermería en el juicio clínico: la valoración y el diagnóstico." Enfermería en cardiología: revista científica e informativa de la Asociación Española de Enfermería en Cardiología 62 (2014): 25-31. 